\title{
A OFERTA DE ATENDIMENTO: ESTRUTURAS DE SAÚDE PARA A CRENÇA NO MEIO URBANO ${ }^{1}$
}

\author{
L'OFFRE DE SOINS: STRUCTURES DE SANTÉ POUR \\ L'ENFANT EN VILLE
}

Stéphane Tessier ${ }^{2}$

TESSIER, S. A Oferta de Atendimento: Estruturas de Saúde para a Criança no Meio Urbano. Rev. Bras. Cresc. Des. Hum. III til ): São Paulo, 1993.

\section{RESUMO}

As estruturas de saúde para as crianças não são organizadas de modo especifico em função do contexto urbano. O exame da literatura mostra que as estruturas modernas, tanto as rurais quanto as urbanas, articulam-se em torno de dois eixos: preventivos (centos de PMI, saúde escolar) e curativo (hospitais ou pediatras privados). Na realidade, a oferta de cuidados de tipo informal, popular ou tradicional é muito mais complexa e o autor descreve as relações entre serviços de saúde e população sob a dupla perspectiva da utilização dos serviços modernos oferecidos e da descrição da procura aos recursos.

\section{RÉSUMÉ}

Les stnuctures de santé pour les enfants ne sont pas orgisées de façon spécifique en fonction du contexto urbain. L'examen de la littérature montre que les structures modernes autant rurales qu'urbaines s'articulent autour de deux axes: preventif (centres de PMI, santé scolaire) et curatif (hôpitaux ou Catres privés). En realité, l'offre de soins informelle, populaire ou traditionnelle est beaucoup plus complexo et l'auteur décrit les relations entre services de santé et population sous la double perspective de l'utilisation des services modernos offerts et la description des itinéraires des recours.

\section{Os textos e as orientações disponíveis no mundo}

O código de saúde pública da França, que organiza o conjunto dos serviços sanitários rurais e urbanos, foi modificado recentemente pela lei de descentralização, mas ainda não temos uma estratégia nova em matéria de saúde urbana da criança. Pelo artigo L150 do código de saúde pública modificado (1 ), as atividades de proteção materno-infantil (PMI) "são organizadas sobre uma base territorial, em função das necessidades sanitárias e sociais da população e segundo normas múnimas fixadas por uma via regulamentar”. Este artigo confirma a noção de "circunscrição da PMI”, deixada para a apreciação dos departamentos: a carta sanitária das divisões das estruturas só pode ser utilizada para distribuir os hospitais privados (2). A nova lei de orientação para a cidade (3) não faz nenhuma alusão ao setor da

1 Este texto representa um resumo da versão negral de mesmo título publicada na Synth $\sim$ se Bibliographique dá publicada na Synthèse Bibliographique du CIE: "Enfamt et Villes: Urbanisme, Santé et Societé”, CIE, Paris, 1992.

2 Pesquisador do Centre International de L’Enfance - Paris. 
saúde, ficando este campo afeito as regulamentações puramente sanitárias e ao setor privado.

Os projetos de desenvolvimento social de bairro (DSB) ou urbano (DSU) para bairros "de risco” têm, por sua vez, dificuldades em integrar a dimensão saúde (4). “A abordagem-saúde pode permitir a emergência de um discurso coletivo dos usuários, que, sem substituir o discurso individual do doente, abre o diálogo com o sistema de atendimento. Porque não se trata de refazer o que outros (instituições profissionais, etc.) já fazem, e geralmente muito bem" (5).

Tudo isto, válido para a saúde do adulto, é ainda mais válido paras a criança que, aparentemente, é bem atendida pelas estruturas existentes. Não há na França politica urbana de saúde especificamente para a criança, do mesmo modo que inexiste outra para a arca rural. Os recursos colocados em prática são urbanizados defacto e parecem ser suficientes para cobrir as principais necessidades da criança. A situação não é diferente na Europa, se julgarmos por uma pesquisa recente sobre "dez bairros europeus em crise" (6), feita em cinco países (Alemanha, Bélgica, França, Holanda e Reino Unido). A saúde está simplesmente ausente desses projetos de desenvolvimento local.

As ações possíveis para melhorar a saúde urbana (adulto e criança) são numerosas. Politicamente, pode-se dividi-las em dois tipos (7). De um lado, uma perspectiva setorial, cujas diretrizes partem de cada ministério especializado (saúde, educação, promoção social); de outro, um "ministério de melhoramento urbano" encarrega-se de coordenar as diferentes ações setoriais. Na França, as experiências de saúde urbana são limitadas a uma escala comunitária e colocadas em prática nos "bairros dificeis" pelos municípios, com apoio da Delegação Interrninisterial da Cidade (8). Mais frequentemente, elas se limitam a ações de diagn ${ }^{\wedge}$ óstic $\wedge \mathrm{O}$ comunitário, seguidos de debates e de tomadas de posição, permitindo que seja criada uma nova dinamica de bairro $(9,10)$.

A Organização Mundial de Saúde (11) definiu vários tipos de obstáculos ligados ao contexto urbano: heterogeneidade das comunidades; dificuldade em atingir "os mais pobres dos pobres", dos quais fazem parte as crianças de rua; impossibilidade de conseguir voluntários numa população que luta para sobreviver; oposição do "stablishment" médico; urbanização incontrolável ...

\section{Estruturas “modernas”}

Apesar de desorganizada e caótica, há uma "nebulosa” de seniços de saúde of erecida à crian- ça nas cidades. Rivais, concorrentes ou complementares, essas estruturas respondem, na prática, a diversas necessidades de saúde. Falar de estruturas de saúde lembra automaticamente dos polos de "excel ncia tecnológica", que são historicamente os hospitais (12). Se os hospitais especializados para crianças ou os serviços hospitalares em pediatria têm uma localização urbana, esta é entao a evidência ligada ao único papel centralizador da cidade.

Às vezes impostas do exterior, como ocorre nos pa ses pós-coloniais (13), esta centralização não dá lugar a nenhuma especialização urbana dos serviços. Suas necessidades respondem àquelas nascidas tanto das patologias urbanas, quanto das rurais. Em alguns contextos particulares, experiências de deslocamento rural de assistência estritamente hospitalar, como a cirurgia, permitiram aliviar certos hospitais (14). Em Xangai, 20\% dos pacientes hospitalizados nas estruturas municipais e $60 \%$ nas especializadas vêm do campo (15).

As composições das estruturas de saúde menos sofisticadas para as crianças, de primeira linha, variam segundo as opções politicas dos pa ses (16). O setor curativo (atendimento das crianças doentes) é concebido geralmente como a base de uma piramide sob a égide de um hospital. Faz parte de sistemas privados de atendimento, quando existem, mas pode também ser integrado no quadro dos centros públicos de saúde matero-infantil englobando mães e crianças de zonas rurais e urbanas. NaFrança, os centros dePMI não têm, teoricamente, o direito de atender doentes, papel que ficapara os centros mutualistas e municipais de saúde, que nem sempre contam com os meios desejados (17) para prestar cuidados de qualidade às crianças desfavorecidas.

O setor preventivo (essencialmente vacinações, distribuição de micro-nutrientes-vitaminas D e A, ferro, exames sistemáticos e tomadas de peso) repousa sobre os mesmos pilares, com um papel mais destacado do que o dos centros de PMI. A essas facilidades especificamente sanitárias, juntam-se as estruturas de saúde escolar, que têmpor objetivo a supervisão da higiene e sobretudo dos controles de saúde obrigatórios.

Sabendo-se que a mortalidade infantil estava sobretudo ligada à má-nutriçao e às diarreias, fizeram-se programas de renutrição e reidratação de crianças. Justificadas pelos seus promotores nas zonas rurais sobre bases epidemiológicas, essas estruturas foram transpostas para numerosos contextos urbanos. Instalaram-se centros emBurkinaFaso (18), no Senegal (19), no 
Camboja (20) e outros países, mas houve problemas. As populações urbanas, "contrariamente ao que se passa no sistema autoritário ou no meio rural (21)”, têm necessidades e comportamentos de saúde imprevisiveis, não ligados à proximidade geográfico (22).

A gratuidade dos serviços curativos e preventivos de primeira linha (ou seu reembolso) é um principio frequentemente utilizado para assegurarumacesso igual à população desfavoreeida na França (23). Isso foi recentemente recolocado em questão nos países em desenvolvimento para permitir uma melhoria do seu funcionamento (24). Assim, o pagamento dos serviços públicos (incluido às vezes o tratamento de crianças, mesmo as atividades preventivas) tem sido posto em prática em numerosos contextos, em particular africanos (25).

\section{Coordenação de estruturas}

Se os problemas de relação entre estruturas de primeira linha e hospitais não são específicos aos serviços pediátricos, eles são importantes na medida que a proteção materna e infantil representa $80 \%$ dos cuidados de saúde primários (26). As estruturas das periferias são frequentemente sub-utilizadas por falta de recursos e de pessoal; já as mais centrais estão sobrecarregadas e são mal utilizadas (11). Os hospitais, conscientes da necessidade de tomar contato com a população, tentam sair de seus muros. Em Cuba, a integração dos médicos de família nas' consultas externas dos hospitais (18) parece ter sido positiva, valorizando o trabalho das estruturas periféricas e dos médicos da cidade.

Nos Estados Unidos, à medida que as doenças infecciosas agudas da criança foram sendo controladas, passou-se de programas essencialmente orientados para medicina, para atividades de origem multifatorial, que requerem apoio de outras disciplinas. A cobertura por vacina em zona urbana caiu nas zonas mais pobres, indo a 50\% das crianças na idade pré-escolar. O "objetivo decisivo" para os EUAnos anos 90 é o de assegurar cuidados prin rinc continlUoc e globais às criara corri neces^ ${ }^{\wedge i} \sim 1$ ade $\sim$ Ele saúde especificas, Cali, na Colômbia, reforçou as estruturas periféricas, insistindo na necessidade de fazer da família a unidade de base dos serviços de saúde (15, p.87). Outros aconselham a população a utilizar os postos de saúde periféricos, e assentar nas favelas estruturas de curas, às quais serão progrcssivamente anexadas atividades preventivas (27).

\section{Programas de proteção}

Alguns planos específicos com base nas estratégias de cuidados primários de saúde (28) $\mathrm{vi}^{\wedge}$ samre $^{\wedge} \mathrm{S}^{\wedge} \mathrm{p}^{\wedge}$ ondera ${ }^{\wedge} \operatorname{pre}^{\wedge} \mathrm{O}^{\wedge} \mathrm{C}^{\wedge} \mathrm{u}^{\wedge} \mathrm{pa}^{\wedge} \mathrm{c}^{\wedge} \mathrm{o}^{\circ} \mathrm{e}^{\wedge} \mathrm{s}$ precisas, essencialmente prevenção e cuidados com a desnutrição, vacinação e reidratação por via oral. Mas, genericamente, as orientações da atenção primáriapara a saúde parecem universalmente necessárias: encorajamento ao aleitamento materno e práticas de desmame apropriadas, acompanhamento do crescimento, luta contra as doenças de base das mulheres e crianças, e uma educação comunitária para a saúde (15, p. 70).

Sempre houve distribuição de alimentos nas escolas ou comunidades para melhorar o estado de nutrição das crianças. Os "copos de leite” peruanos (29) eram similares às distribuições de leite achocolatado nas escolas francesas dos anos 50. As vezes, essas distribuições podem ter consequências surpreendestes, como no Rio de Janeiro, onde as mães trocavam o leite que recebiam por pão branco, ao qual atribuíam erroneamente maior valor nutritivo (30).

Os serviços mantém complexas relações com a população, sempre difíceis de serem compreendidos e avaliados. Dispomos de diversas abordagens, sejam diretas (indicadores de cobertura ou estado de saúde), sejam indiretas, de utilização de serviços, até de estudos de demanda Os serviços repousam em indicadores sempre discutíveis, em particular na cidade. Num bairro de São Paulo (31), quando a cobertura vacinal está relativamente boa e homogenea, as consultas apresentam consideráveis diferenças sócio-econômicas. EmMadras, Índia, os programas de suplementação alimentar e micro-nutrientes têm números muito inferiores aos números de cobertura vacinal, com somente $15 \%$ de famílias se beneficiando de tais programas (32). Muitos autores ressaltam que os indicadores de saúde urbanos não refletem sempre a situação do conjunto da população, mesmo porque os habitantes dos barracas não aparecem nas estatísticas.

\section{Utilização dos serviços}

Em Porto Alegre, um estudo mostrou que 37\% das consultas de cuidados primários de saúde foram consagradas às crianças com menos de dez anos (33). Para menores de cinco anos, este número caiu para25\% num meio urbano da África do Sul, e subiu para 43\% na periferia de Dakar (33). Outrapesquisa, realizadaem Londres, sobre crianças de dois anos, com base nos dossiês dos visitadores (quadro 1) (34), revela que 20\% das 
consultas de urgências na região central são ligadas a problemas médicos não infecciosos, contra 1,ó\% da periferia. Atribui-se a diferença a uma percepção mais forte na cidade do fato de as urgências serem 'bm modo mais rápido de se conseguir a opinião de um especialista que trabalha num centro prestigiado".

\begin{tabular}{cccc}
\hline $\begin{array}{c}\text { Residência } \\
\% \text { que teve }\end{array}$ & $\begin{array}{c}\text { Londres Centro } \\
(\mathrm{n}=756)\end{array}$ & $\begin{array}{c}\text { Periferia } \\
\text { Próxima } \\
(\mathrm{n}=127)\end{array}$ & $\begin{array}{c}\text { Periferia } \\
\text { Longínqua } \\
(\mathrm{n}=96)\end{array}$ \\
\hline $\begin{array}{c}\text { Pelo menos } \\
\text { uma admissão }\end{array}$ & $28,4 \%$ & $23,6 \%$ & $14,4 \%$ \\
\hline $\begin{array}{c}\text { Pelo menos uma } \\
\text { consulta externa } \\
(6 \text { ou mais }\end{array}$ & $40,5 \%$ & $22 \%$ & $20,6 \%$ \\
\hline $\begin{array}{c}\text { Pelo menos uma } \\
\text { consulta urgente } \\
\text { (idade média }\end{array}$ & $26,6 \%$ & $2,4 \%$ & $5,2 \%$ \\
\hline
\end{tabular}

Quadro 1: Porcentagem de crianças residindo no centro e arredores de Londres que utilizaram certos serviços (em qualquer localização) nos seus dois primeiros anos

Na Arábia Saudita, descobriu-se que adultos e crianças têm tendência idêntica de fazer consultas no hospital, apesar de existirem estruturas primárias adequadas (35). As sugestões feitas para melhorar a situação deixam em mente a necessidade de aumentar o número de pessoal qualificado e de médicos nas estruturas primárias (34\%), seguindo-se uma melhora dos serviços. A utilização de serviços de saúde por crianças nas proximidades do domicilio é de falo a situação mais frequente.

Nas zonas urbanas e rurais, a utilização de serviços preventivos varia segundo o tipo de serviço e nivel sócio-econômico.

As diferenças reveladas entre vacinações e consultas (31) estão ligadas aos métodos de pres- tação de cuidados: as primeiras, muito ativas e voluutaristas, ao passo que as scgundas nem sempre pe milcm o acesso por causa de dificuldades financeiras (36). Mas na Nigéna, apesar da gratuidade, venfica-se o mesmo afunilamento sócio-econômico na frequência das consultas de prevenção, indo de $30 \%$ das crianças de baixo poder aquisitivo a $62 \%$ das crianças de nivel mais alto, que tiveram pelo menos uma visita (37).

O autor do trabalho londrino descobriu que as diferenças determinantes do seguimento das consultas preventivas pediátricas dependem de condições sociais, econômicas e de hábitos. As mães muito jovens traduzem sua inquietude por uma utilização maior das consultas (mais de 11 consultas no primeiro ano da criança), ao passo que as mães com mais de 36 anos não comparecem com frequência.

\section{A Demanda aos Serviços}

Emmatéria de saúde da criança, a primeira a decidir sobre o recurso a ser procurado é a mãe ou tutor: por isso, uma perspectiva de estudo das relações entre a popular e c Diadema de saúde pode ser 0 estudo das modalidades e determinantes desses recursos. A primeira forma de tratamento geralmente é familiar e próxima da criança, tanto por razões culturais como económicas. Um estudo no Rio de Janeiro conclui que são sempre as mulheres que decidem primeiro a forma de enfrentamento dos problemas de saúde (30). O primeiro tratamento é, portanto, a au omedicação, que, apesar de mais frequente na zona rural, está presente nos meios urbanos.

Em Ouagadougou (38), um estudo efetuado com familias acusou a utilização de diferentes recursos terapêuticas, dependendo da idade do paciente (quadro 2). A mais baixa porcentagem de abstinência foi encontradaemrecém-nascidos. Além do forte investimento cultural feito sobre este grupo etário, é provável também que o custo

\begin{tabular}{|c|c|c|c|c|}
\hline & Automedicação & Abstenção & Curandeiro Tradicional & Sistema Moderno \\
\hline \multicolumn{5}{|l|}{ Ouagadougou] } \\
\hline $1989[26]$ & $56 \%$ & $12 \%$ & $2,4 \%$ & $30 \%$ \\
\hline Adultos & $34 \%$ & $9 \%$ & $3 \%$ & $53 \%$ \\
\hline Crianças < 1 & $58 \%$ & $9 \%$ & $1,5 \%$ & $51 \%$ \\
\hline \multicolumn{5}{|l|}{ Crianças 1-14 anos } \\
\hline \multicolumn{5}{|l|}{ Pikine 1985 [68] } \\
\hline Mulheres & (não estudado) & $22 \%$ & (não estudado) & $78 \%$ \\
\hline Crianças & & $23,3 \%$ & & $78,7 \%$ \\
\hline
\end{tabular}

Quadro 2: Recursos terapêuticos quando de um episódio de doença 
das receitas, bem menos onerosas que para as outras categorias da população, desempenhe um papel importante neste fenômeno.

Certos serviços de saúde exigem diretamente das familias que recolham informações sobre o estado de saúde das crianças, como em Manila, Filipinas (15, p.64). Em Jacarta, 200 mil crianças das escolas receberam formação para serem “jovens médicos” dos cuidados primános de saúde (15, p. 25).

Os elementos importantes dessa tomada de decisão repousam na acessibilidade geográ^^fica e económica dos serviços, mas são também culturais. Todos dependem da patologia declarada pela criança, e não existe comportamento estereótipado.

O fenômeno de distribuição sócio-econômico da frequência das consultas depende dos recursos que a população está pronta a destinar à Saúde, e do acesso e disponibilidade dos serviços privados. Em Khartum, Sudão (39), 66,9\% das populações mais abastadas utilizam os seviços particulares, enquanto os pobres da Nigéria (40) raramente o fazem as proporções são de 46,2\% contra 7,2\%. Em Pikine, Senegal, as proporções são ainda mais fracas: 19,3\% contra 7,2\%. No Irã (41), as crianças eram vacinadas de maneira diferente, dependendo da área. Em Teerã, 25\% são vacinadas pelo setor privado, contra $0,7 \%$ em outras cidades iranianas e $0,04 \%$ na zona rural.

Mas a parcela que a população está disposta a dispender em consulta privada varia segundo a idade dos pacientes e suas patologias. $\mathrm{O}$ estudo feito em Pikine (42) revela este fenómeno (quadro 3). Os autores, analisando diferentes causas de consulta de cada categoria, descobriram que as doenças ginecológicas nas mulheres e o sarampo para as crianças, dão lugar a menos abstenções e demonstram também à importância das percepções culturais das patologias na escolha do recurso de que se lança mão.
A influência da idade sobre os recursos foi mostrada por uma pesquisa nacional de nutrição e de saúde no Peru (43). Esta pesquisa distingue os "resignados", que percebem sozinhos (ou por seus próximos) uma patologia, mas não se consultam. Eles são encontrados em meio às crianças de um a quatro anos, para as quais 60\% dos episódios revelados não deram lugar a uma consulta. No oposto, o grupo mais sensível são os adolescentes de 15 a 24 anos, que só se abstêm em $1 \%$ dos casos.

Constatou-se também que a difusão de informações e de conhecimentos seguem as mesmas divisões sócio-econômicas, e que os diferentes recursos não são explicáveis somente pelos argumentos económicos. A automedicação depende dos conhecimentos, modernos e tradicionais, que a população tem em matéria de terapêutica. Em Chandigar, India (44),61\% dos pais urbanos (40\% de baixo nivel sécio cultural, 47 e 93\% nas categorias mais abastadas) dão antitérmicos em casa para cada febre de suas crianças (27\% na zona rural). A diarréia é outra circunstância para automedicação pelas mães: em Bangkok, no caso de diarréias, 54\% das mães ficavam em casa (37\% pediam sais de reidratação aos pesquisadores e $17 \%$ tinham outros medicamentos).

O “trajeto-padrão” das diarréias em Pikine (45) começa pela automedica,cão; não havendo melhora, é então continuado por tratamento mudemos. Os conhecimentos não são somente de ordem terapêutica, mas também administrativa, como mostram estudos na $\mathrm{F}^{\wedge}$ Mnça. Além desses conhecimentos, o papel da expressão ou afirmação social que pode ter a doença (de adulto ou criança) é ressaltada pelos estudiosos. Nos bairros mais desfavorecidos da França, “a doença é o último refúgio no qual o individuo encontra reconhecimento social e reconstrói sua realidade” (4). Está claro que as crianças exercem frequentemente o papel de transferência da dessocialização dos

Quadro 3: Frequência de recursos segundo o tipo e o custo dos aios

\begin{tabular}{|c|c|c|c|c|c|c|c|}
\hline \multicolumn{2}{|c|}{$\begin{array}{l}\text { Local do } \\
\text { Recurso em Pikine, Abstenção } \\
1985 \text { Custo [68] }\end{array}$} & $\begin{array}{c}\text { Díspensário } \\
\text { Público }\end{array}$ & $\begin{array}{c}\text { Díspensário } \\
\text { Religioso }\end{array}$ & $\begin{array}{c}\text { Díspensário } \\
\text { Empresas }\end{array}$ & $\begin{array}{l}\text { Hospital } \\
\text { Público }\end{array}$ & $\begin{array}{l}\text { Clínicas } \\
\text { Privadas }\end{array}$ & Outros \\
\hline \multicolumn{8}{|l|}{ Custo em Francos } \\
\hline Franceses & 0 & 3 & 5 & 0 & 3 & 80 & 1 \\
\hline \multirow{3}{*}{$\begin{array}{c}\text { \% de todos os } \\
\text { recursos em casos } \\
\text { de Patologia da mãe } \\
\text { Patologia da criança }\end{array}$} & & & & & & & \\
\hline & 22 & 44 & 7 & 4 & 9 & 10 & 4 \\
\hline & 23 & 52 & 15 & 2 & 3 & 3 & 1 \\
\hline
\end{tabular}


pais, fazendo aparecer “a medicina como um mecanismo capaz de tecer ligações entre o indivíduo e a sociedade” (4). Frente a esta situação, a "única porta de entrada no sistema de saúde são os serviços de urgência dos hospitais, umlocal onde não se pode encontrar a cura quando não se vem por uma urgência” (23).

\section{Conclusão}

FOM OS aspectos de tecnologia pediátrica, o atendimento da saúde da criança em zona urbana na França vive uma tradição de proteção social solidamente estabelecida, cujas origens são antigas e pouco questionadas. Em consequência, a ausência de uma polltica estruturada da matéria é compensada pela existência de uma miríade de estruturas, instituições e pessoal, que, mesmo pouco coordenados e não raro rivais, acabam constituindo uma rede de ajuda e de atendimento bastante densa, da qual poucas crianças podem escapar.

\section{Bibliograi;a e Notas}

01. Lei ${ }^{\circ} 89.899$ de 18/12/89 publicada no J.O. de 19112189

02. Lei de $31 / 12 / 1970$

03. Lei $n^{\circ} 91.882$ de 13/07/1991 publicada no J.O. de 19/07/1991, retificada em 29/10/1991

04. BERTOLOTTO, F. Santé publique et crise urbaine dans les quartiers défavorisés; du souci de santé à l'enjeu démocratique; Lumières de la Ville; Juin 1992; n 5; pp. 5767

05. REY,M. Dossier politiques de santé; Revue Fnsemhles; Délégation Interministérielle à la Ville;Mars 1992;n 35; p.l 1

06. JACQUIER, C. Voyage dans dix quartiers européens en crise l'Harmattan, Paris 1991.

07. HARPHAM, T.; STEPHENS, C. Policy directions in urban health in developing countries - The slum improvement approach; Soc. Sci.Med.; 1992; 35; 111-20.

08 Des nombreuses expériences ont été menées mais rares sont celles qui ont donné lieu à une publication accessible. C'est la raison pour laquelle elles ne sont pas citées de façon exhaustive ici.

09. BRIXI, O.; FEBVREL, D. Diagnostic communautaire dans une cité de la banlieue parisienne: la démarche entreprise. In: Santé communautaire; Concepts, action, formation; CIE, Paris 1990; 57-67.

10. BAUMMANN, M. Promotion de la santé au sein d'une communauté urbaine; A propos de
Mas o que é verdade para a França e outros países, que aliam potência econômica e tradição social, não é regra nas nações em desenvolvimento e até mesmo nos Estados Unidos, segundo demonstram vários autores. Frente à emergência dos problemas, parece agora necessáno e urgente formular políticas urbanas de saúde. Será preciso ainda, na definição de modelos de desenvolvimento urbano, que, à eficiência, até agora privilegiada, sejam explicitamente anexados componentes sanitários sociais.

A complexidade das determinantes do acesso aos cuidados, como é narrada na literatura especializada, impõe que as reflexões sobre a acessibilidade das crianças aos cuidados transponha o quadro apenas financeiro. Elas devem também abordar questões de legitimidade das estruturas, da representação cultural da doença, da "utilização" social das patologias, etc. Isso para que o sistema de saúde destinado às crianças se torne um ator efetivo no desenvolvimento urbano e não simplesmente um prestador de seniços.

la ville de Toul (Lorraine). In: Santé communautaire; Concepts, action, formation; CIE; Paris 1990. 67-78.

11. OMS. Improving urban health system; Rap. Trim. Stat. San. Mond.; 1991; 44; 234-40.

12. OMS. Hôpitaux et santé pour tous; Rapports techiniques no 744; OMS, Genève, 1987.

13. MUGANZI, Z.; OBUDHO, R.A. The spatial distribution of health services in the urban centres of Kenya. In: Urbanization et santé dans le Tiers Monde; Colloque ORSTOM, Paris, 1989, 235-55.

14. SEKOU SAKO, A. La chirurgie "foraine" au Niger: 12 années d'expérience; La place et le rôle de l'hôpital en Afrique; Actes des Journées Internationales d'Ahidjan, 6-8 Juin 1990; Acodess, Paris 1990; pp. 91-95.

15. OMS; TABIBZADEH, I.; ROSSI-ESPAGNET, A.; MAXWELL, R. Pleins feux sur les villes, Améliorer la santé dans les villes du Tiers Monde; 183p.; OMS Genève, 1991.

16. Des nombreux documents détaillent les systèmes de santé de chaque pays. En France citons: BRUCKER, G.; FASSIN, D. et al.; Santé Publique; Ellipses Paris 1989. LEVY, A. et al.; Manuel de santé publique; Masson 1989. MONNIER, J.; DESCHAMPS, J.P.; FABRY, J., MANCIAUX, M.; RAIMBAULT, A.M.; A.M. Santé Publique santé de la communauté; Simep, Villeurbanne, 1980.

17. BEAUPIN, A. (Editorial) Le nouveau Centre de Santé; Juillet-Aoüt, 1992; nº 98 (Ed. Union 
Syndicale des médecins de centres de santé).

18. Centres CREN sur l'ensemble du pays

19. FONTAINE, O.; BEAU, J.P.; BRIEND, A. Un centre expérimental de rehydratation et de réhabilitation nutritionnelle; In: Urbanisation et santé dans le Tiers Monde; Colloque ORSTOM, Paris 1989; 455-61.

20. Centres RINE à Phnom Penh

21. JEANNEE, E.; FERNEA, L.A. Information sanitaire et statistiques demorbidité enmilieu urbain africain; In: Urbanisation et santé dans le Tiers Monde; Colloque ORSTOM, Paris, 1989; 433-42.

22. LALOE, F.; SALEM, G.; BENARD, C. Dimensions géographiques de la couverture sanitaire à Pikine. In: Urbanisation et santé dans le Tiers Monde; Colloque ORSTOM, Paris, 1989; 471-ó.

23. MEDECINS sans frontières: Mission solidarité France; Rapport Annuel, 1991.

24. BANQUE MONDIALE: Financing Health Services in Developing Countries, An agenda for refomm; A World Bank Policy Study Washington, D.C., 1987; repris par l'UNICEF sous le terme d" nnitiative de Bamako".

25. UNICEF. Financement communautaire des services de santé locaux: expériences acquises en Afrique; UNICEF, New York, 1989.

26. Le CIE mène actuellement une étude multicentrique avec l'Institut de Médecine Tropicale d'Anvers sur les déterminants des recours aux soins pour les enfants en France, Togo, Congo, Algérie et Maroc.

27. HARPHAM, T. Urbanisation and Primary Health Care; In: Urbanisation et santé dans le Tiers Monde; Colloque ORSTOM, Paris, 1989; 363-ó.

28. OMS. Les soins de santé primaries; Déclaration d'Alma Ata; OMS;

29. Depuis plus de dix ans, un vaste programme est organisé par plusieurs organisations non gouvernementales de Lima. Chaque enfant scolarisé doit avoir un verre de lait par jour.

30. NOVAES DA MOTA, C.; NEIVA, V.C. Jardim Primavera: applications of anthropological procedures to the assessment of programmes of nutrition and primary health care in a suburban community of Rio de Janeiro; Food and Nut. Bull.; 1990; 12; 313-7.

31. MONTEIRO, C. A.; GUERRA MEDINA, M. C.; D’AQUINO BENÍCIO, M. H.; MEYER, M. Estudo das condições de saúde das crianças do município de São Paulo (Brasil), 1984/ 85: IX Cobertura e qualidade da assistência materno-infantil; Rev. Saúde Publ., São Paulo, 1988; 22; pp. 179-8.

32. SRILATHA, V.; AITKEN, I.W. A health risk index for assessing PHC coverage in urban India; Health Policy and Planning; 1991, 6; pp. 234-43.
33. RADAELLI, S. M.; TAKEDA, S.M.P.; GIMENO, L. I. D.; WAGNER, M. B.; KANTER, F.J.; DE MELLO, V.M.; BORGES, J. C.; DUNCAN, B.B. Demanda de serviço de saúde comunitária na periferia de área metropolitana; Rev. Saúde Públ., São Paulo, 1990; 24; 232-40.

34. WHILE ALISON, E. Early Childhood Morbidity in Three Contrasting Urban Districts; Public Health; 1988; 102; 135-9.

35. ABU ZEID, H. A. H.; Health Services utilization patterns of two urban communities in Abha, Saudi Arabia; Jour. Com. Health; 1989; 14; pp. 65-77.

36. ADEDOYIN, M.A.; WATTS, S.J. Child Health and Child Care in Okelele: an indigenous arca of the city of Ilorin, Nigeria; Soc. Sci. Med.; 1989; 29; pp. 1333-41.

37. RAYNAUT, C. Disparités économiques etinégalités devant la santé a Maradi (Niger); In: Urbanisation et santé dans le Tiers Monde; Colloque ORSTOM, Paris, 1989; 477503.

38. DEVELAY, A. Utilisation des soins de santé em milieu urbain au Burkina Faso; Résultat d'une enquêteparinterrogatoire deménages dans la ville de Ouagadougou; Thèse; Paris, Heidelberg 1991.

39 HERBERT, D.T.; HIJAZI, N.B. Ill-health and health care in Khartoum/Omdurman; Soe. Se. Med.; 1984; 18; 335-43.

40. UYANGA, Y. Rural-urban migration and sickness/health care behavior: a study of Eastern Nigeria. Soc. Sci. Med.; 1983; 17; 579-83.

41. NASSERI, K.; SADRIZADEH, B.; MALEKAFZALI, H.; MO HAMMAD, K.; CHAMSA,M.; CHERAGHCHI-BASHI, M.T.; HAGHGOO, M. and AZMOODEH,M. Primary Health Care and Immunisation in Iran; Public Health; 1991; 105; 229-38.

42. FASSIN, D.; JEANNEE, E.; CÉBE, D.; REVEILLON, M. Who consults and where? Socio-cultural differentiation in access to health care in urban Africa; Tritern. I. Epi..; 1988; 17; pp. 858-64.

43. ANSSA-PERU: Analisis dei sector salud; Perfil de salud de la poblacion peruana; Informe technico $\mathrm{N}^{\circ}$ 1; Lima, 1986.

44. SINGHI, S.; PADM I, P.; SOOD, V.: Urban parents' understanding of ferver in children: its dangers and treatment pratice; In ! i Pediatrics; 1991; 28; pp.501-5.

45. WERNER, J. F. Du symptôme au système: une exploration anthropologique des diarrhées du ^Jeune à Pikine. In: Urbanisation t santé le Tiers Monde; Colloque ORSTOM, Paris 1989; 347-53. 